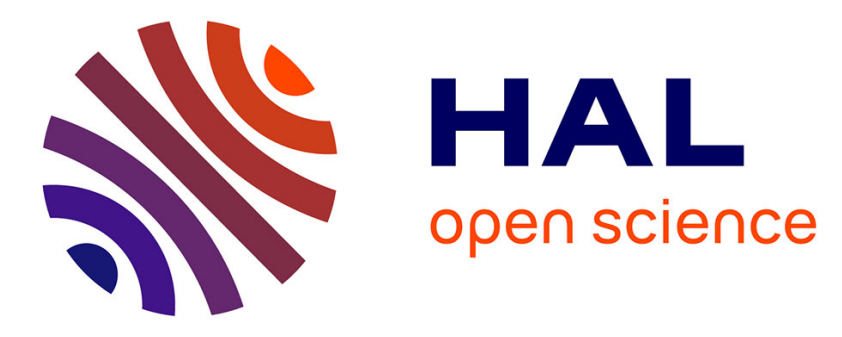

\title{
Experimental observation of the emergence of Peregrine-like events in focusing dam break flows
}

Frédéric Audo, Bertrand Kibler, Julien Fatome, Christophe Finot

\section{To cite this version:}

Frédéric Audo, Bertrand Kibler, Julien Fatome, Christophe Finot. Experimental observation of the emergence of Peregrine-like events in focusing dam break flows. Optics Letters, 2018, 43 (12), pp.28642867. 10.1364/OL.43.002864 . hal-01738530

\section{HAL Id: hal-01738530 \\ https://hal.science/hal-01738530}

Submitted on 20 Mar 2018

HAL is a multi-disciplinary open access archive for the deposit and dissemination of scientific research documents, whether they are published or not. The documents may come from teaching and research institutions in France or abroad, or from public or private research centers.
L'archive ouverte pluridisciplinaire HAL, est destinée au dépôt et à la diffusion de documents scientifiques de niveau recherche, publiés ou non, émanant des établissements d'enseignement et de recherche français ou étrangers, des laboratoires publics ou privés. 


\title{
Experimental observation of the emergence of Peregrine-like events in focusing dam break flows
}

\author{
Frederic Audo, Bertrand Kibler, Julien Fatome, Christophe Finot
}

Laboratoire Interdisciplinaire Carnot de Bourgogne, UMR 6303 CNRS Université Bourgogne Franche-Comté, 9 av. Savary, 21078 Dijon, France *Corresponding author: christophe.finot@u-bourgogne.fr

\begin{abstract}
Simple photonic fiber-based workbenches have been able to emulate well-known nonlinear wave dynamics occurring in deep- or shallow- water conditions. Here, by investigating the nonlinear reshaping of a flattop pulse upon propagation in an anomalous dispersive optical fiber, we observe that typical signatures of focusing dam break flows and Peregrine-like breather events, can locally coexist into spontaneous pattern formations. Experimental measurements are in good agreement with our numerical predictions.
\end{abstract}

OCIS codes: (190.4370) Nonlinear optics, fibers; (060.5530) Pulse propagation and temporal solitons;

Optical fibers have shown their incredible ability to act as a perfect testbed platform to explore the richness of the dynamics of nonlinear physics. Indeed, their weak level of losses as well as the excellent knowledge of both linear and nonlinear physical characteristics are key ingredients to carry out experimental demonstration of new coherent solutions with an outstanding agreement with the theoretical solutions of the nonlinear Schrödinger equation (NLSE). As soon as the early 80s, solitons were demonstrated in single-mode optical fibers [1]. In the $21^{\text {st }}$ century, new kinds of solitons relying on a continuous wave background have stimulated a large experimental interest, especially in the context of the rogue-like structures emerging in the anomalous dispersive regime of propagation. Taking advantage of the advanced control provided by the components of the telecommunication industry and new ultrafast characterization methods, Peregrine wave [2,3], higher-order or superregular breathers $[4,5,6]$ have been experimentally generated. Recent other works have stressed the crucial importance and universality of such structures, especially the Peregrine wave $[7,8,9]$ which can also be detected in deep water and other nonlinear medium governed by the NLSE $[10,11]$. Normally dispersive fibers have also stimulated experimental research, mainly driven by the interest in the study of dispersive shock waves (DSW) [12] : optical equivalent of undular bores [13], reproduction of dam-breaking problems [14], or the Riemann simple waves [15], have provided recent examples of the insights that can be obtained in the defocusing regime of propagation.

Nevertheless, nonlinear dynamics observed in the anomalous or (weak) normal dispersion regimes of propagation are most of the time regarded as two completely different cases. The first one is dominated by bright soliton-like structures and modulationinstability in perfect analogy to deep-water conditions [16]. The second one is ruled by DSWs that satisfy the so-called nonlinear shallow water equations [17]. However, recent theoretical works have stressed that some similar characteristics with the DSW may appear in the regime of focusing nonlinearity with weak dispersion, thus leading to the emergence of dispersive dam break flows in the NLSE box problem $[18,19]$. In this new scenario, the emergence of a nonlinear wave train regularizes an initial sharp transition between the uniform plane wave and the zero-intensity background. Theoretical solutions essentially describe a modulated soliton train. This phenomenon provides a new semi-classical interpretation of that has been previously described in the spatial domain as a nonlinear Fresnel diffraction [20]. This box problem (i.e., an initial square profile) then gives rise to two such counter-propagating modulation dynamics, whose interaction may turn into a cluster of breathers [19]. In the present contribution, we confirm theoretical predictions of Refs. $[18,19]$ by providing a detailed experimental observation of the regularization of sharp transitions from a superGaussian pulse in the presence of focusing nonlinearity. We evidence transient breathing dynamics of dispersive dam break flows in the form of Peregrine-like breather structures. We also characterize the interaction event of the two counter-propagating dispersive dam break flows.

We investigate the nonlinear propagation of a flattop pulse in an anomalous dispersive fiber. The evolution of the slowly varying envelope $A(z, t)$ of the complex electric field is ruled by the focusing NLSE: $i \partial A / \partial z-\frac{1}{2} \beta_{2} \partial^{2} A / \partial t^{2}+\gamma|A|^{2} A=0$, where $\beta_{2}$ is the second order dispersion and $\gamma$ is the Kerr nonlinearity, $t$ and $z$ being the temporal coordinate and the propagation distance respectively. In order to illustrate the typical evolution of the box problem in the regime of focusing nonlinearity with small dispersion, we base our numerical simulations on the fiber parameters used in the experiments discussed below: $\beta_{2}=$ $-21 \mathrm{ps}^{2} \cdot \mathrm{km}^{-1}, \gamma=1.3 \mathrm{~W}^{-1} \cdot \mathrm{km}^{-1}$. We analyze the propagation of an input pulse corresponding to a $12^{\text {th-order }}$ super-Gaussian with a full width at half maximum: $T_{b o x} \sim 600 \mathrm{ps}$ and an input power $P_{0}$ of $2 \mathrm{~W}$. Such a choice of parameters ensures that the nonlinearity initially dominates the dispersive contribution as assumed in Refs. [18,19] to apply the Whitham modulation 
theory. This condition corresponds to $N=\sqrt{L_{D} / L_{N L}} \gg 1$, where $L_{N L}=\left(\gamma P_{0}\right)^{-1}$, and $L_{D}=T_{b o x}^{2} /\left|\beta_{2}\right|$ [23]. Longitudinal evolution of the temporal profile of the pulse is depicted in Fig. $1 \mathrm{a}$.

Upon a first stage of propagation dominated by self-phase modulation $(z<3 \mathrm{~km})$, the pulse develops a strong frequency chirp in its sharp edges. Then, the anomalous dispersion leads to the formation of a highly oscillating pattern induced by the beating between the highly chirped edges and the flattop region of the pulse. In the focusing regime, this sinusoidal beating then evolves into the instantaneous formation of modulated wave trains after $3.75 \mathrm{~km}$ regularizing the initial discontinuities in both edges of the pulse (see inset in Fig. 1a) [18,19]. Such dispersive dam break flows expand inside the pulse with a constant velocity $v= \pm \sqrt{L_{N L} /\left(8\left|\beta_{2}\right|\right)}$ during propagation and with decaying amplitudes of the oscillations. Here we also clearly note that each oscillation of the generated modulated wave presents successive growth-decay cycles, which is a key feature of breather solutions to the focusing NLSE. Inspired from the recent confirmation of the universal emergence of the Peregrine soliton (PS) in the focusing dynamics near a gradient catastrophe [7], we checked their temporal profile at the point of each maximum compression by using the Peregrine profile: $A_{P B}(t)=A_{0}\left[1-4 /\left(1+4\left(t-t_{C}\right)^{2}\left(\left|\beta_{2}\right| /\left(\gamma A_{0}^{2}\right)\right)\right)\right]$. Two examples for $z=3.75$ and $10 \mathrm{~km}$ are reported in Fig. $1 \mathrm{~b}$ for which we observe a very good agreement between these lateral intensity oscillations and the Peregrine solution. The properties of the central peak and adjacent zero-intensity points are wellreproduced by adjusting the parameters $A_{0}$ and $t_{c}$ using the values of the peak power $P_{m}$ and temporal position of the localized structure emerging at the point of maximum compression : $A_{0}=$ $\sqrt{P_{m}} / 3$. For instance, the first Peregrine-like structure reaches a maximum compression at $z=3.75 \mathrm{~km}$ and $t_{C}=276 \mathrm{ps}$. In this particular case, the corresponding value $A_{0}$ is almost equal to the square-root of the power of the flattop pulse taken at $t_{C}$ in the early stage of propagation, namely at $z=1.5 \mathrm{~km}$

When looking at the spectral properties in Fig. 1c, we also confirm the typical features of the Peregrine breather at $z=3.75$ $\mathrm{km}$, in particular the linearly decreasing wings when plotted in a log scale. One can also notice in the magnified view the sinusoidal over-modulation of the spectrum linked to the interference between the two Peregrine-like structures that are temporally spaced by $\sim 554 \mathrm{ps}$. Let us stress here that contrary to the case of smooth pulses where it has been rigorously demonstrated that PS emerge in the semi-classical limit of the NLSE [21], there is up no mathematical proof of the existence of this perfect shape in the dam break problem. Indeed, from the phase-profiles plotted in Fig. 1(b), we can see that even if the typical $\pi$-phase shift of PS is observed in the wings, the phase may also exhibit a significant additional tilt. These conclusions may be confirmed by the use of more advanced tools such as the local inverse scattering transform [22]. The increasing complexity of the temporal waveform does not allow such a spectral comparison at $z=10 \mathrm{~km}$. All the oscillations of the dispersive dam break flows appear to breathe and locally reach the asymptotic Peregrine-like solution at maximum compression. However, their maximal peak power decreases with propagation until reaching an asymptotic state predicted in Refs. [18,19], namely a modulated soliton train with a peak power close to $4 P_{0}$. This can be observed for longer propagation distance $(>15 \mathrm{~km})$ but a detailed study of this phenomenon is beyond the scope of this work.
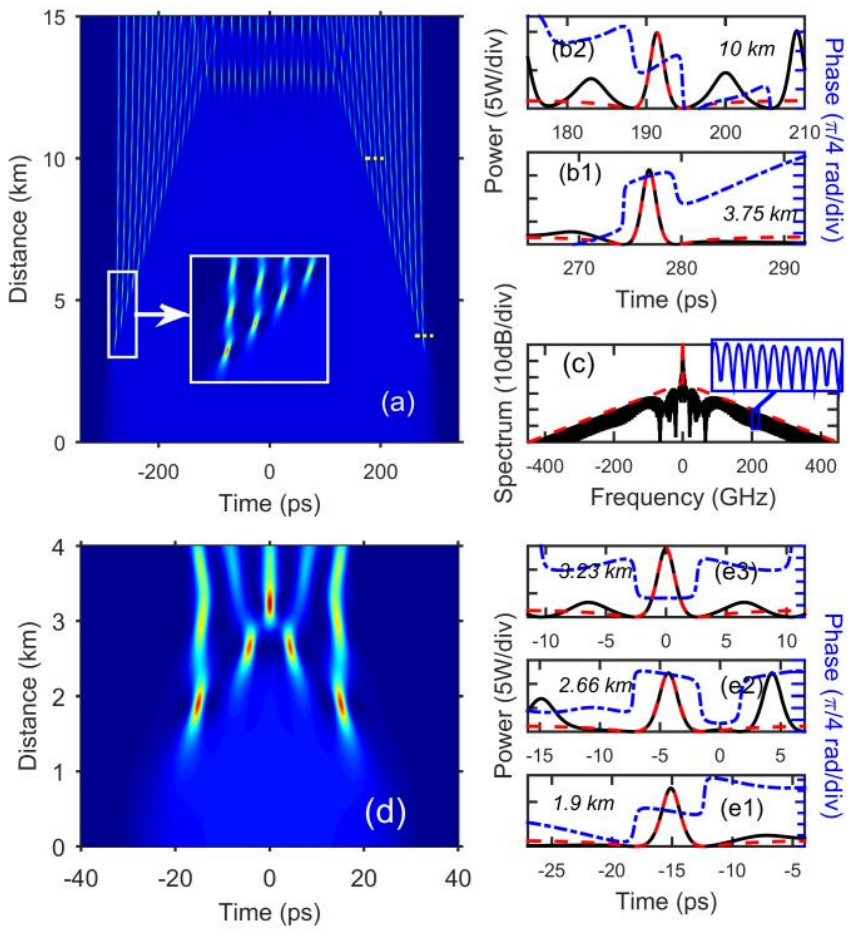

Fig. 1. (a-c) NLSE simulation of the nonlinear propagation of a 600-ps $12^{\text {th-order }}$ super-Gaussian pulse in an anomalous dispersive fiber $(N \sim 210)$. (a) Temporal dynamics as a function of propagation distance. (b) Zoom on different temporal profiles (black solid lines) at 3.75 and $10 \mathrm{~km}$ (bottom to top) compared to the theoretical fit based on Peregrine breather solution (red dashed line). Temporal phase profiles are also plotted with mixed blue lines. (c) Corresponding spectral profile at $3.75 \mathrm{~km}$ (the inset is a magnification of the wing of the spectrum). (de) NLSE simulation for a 52-ps 3rd-order super-Gaussian pulse $(N \sim 52)$. (d) Temporal dynamics as a function of propagation distance. (e) Zoom on differenttemporal profiles (black solid lines) at 1.9, 2.66, and $3.23 \mathrm{~km}$ (bottom to top) compared to the Peregrine solution (red dashed line). Temporal phase profiles are also plotted with mixed blue lines.

It is worth mentioning that, in our simulations, spontaneous modulation instability (MI) emerges mainly from the center of the box problem after $12 \mathrm{~km}$ of propagation (see Fig. 1a). Therefore, the observation of dispersive dam break flows and their possible collision may be limited by the unstable nature of the flat top of the pulse [19]. Indeed, the MI develops from numerical noise and prevents the genuine analysis of the interaction event of the two counter-propagating dispersive dam break flows which should occur at a longer distance $v T_{\text {box }} / 2 \sim 14.4 \mathrm{~km}$ (relation given for the ideal box problem). This noise-driven MI regime is characterized by the emergence of Akhmediev breather structures with frequency that experiences the highest MI gain [24]. However, this spontaneous emergence of MI leads to collision processes with DSW-like nonlinear wave trains, which generate rogue wave structures with peak power up to $29 \mathrm{~W}$. By considering typical experimental input noise in our simulations, the deleterious effect of MI would arise even at shorter distances. Consequently, 
we have reduced the initial pulse width to 52 ps in the experiments in order to enable the interaction of the counterpropagating wave trains on a very short distance. Figure $1 \mathrm{~d}$ presents the corresponding numerical evolution with input pulse used in the experiment. We are able to characterize focusing dispersive dam break flows whose localized peaks are well fitted by the Peregrine breather (see Fig. 1e). In addition, the collision of the two counter-propagating flows can be now easily observed at $3.23 \mathrm{~km}$. We confirm that the resulting high amplitude structure profile is again well fitted by the central peak of the Peregrine solution and associated phase jumps.

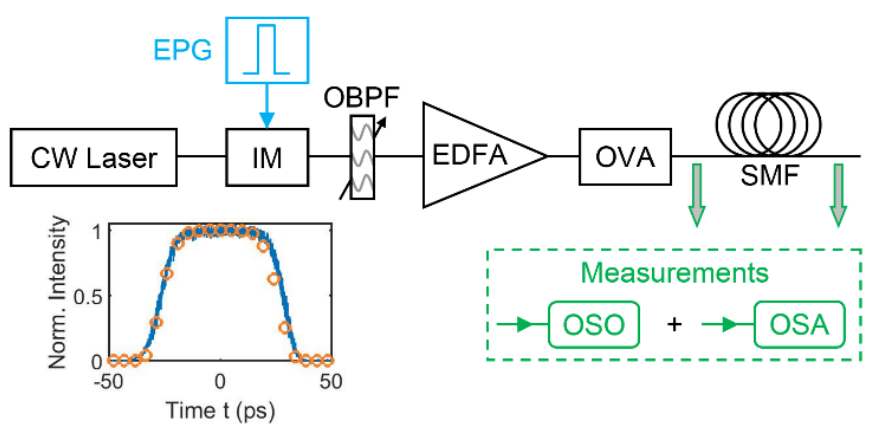

Fig. 2. Experimental setup. CWL: Continuous Wave Laser; IM: Intensity Modulator (40 GHz typical bandwidth); EPG: Electrical Pulse Generator (IXBlue Photonics Modbox); SMF: single-mode fiber; OVA: Optical Variable Attenuator; EDFA: Erbium Doped Fiber Amplifier; OSO: Optical Sampling Oscilloscope (Alnair Labs, Eye-checker EYE 2000C); OSA: Optical Spectrum Analyzer (Yokogawa AQ6370). Inset: temporal profile of the input pulse (solid line) compared with a $3^{\text {rd }}$ order super-Gaussian fit (circles).

The experimental setup is depicted in Fig. 2 and is based exclusively of devices commercially available and typical of the telecommunication industry. A continuous wave laser at 1550 $\mathrm{nm}$ is first intensity modulated using a lithium niobate modulator driven by an electrical generator, thus delivering super-Gaussian pulses at a repetition rate of $100 \mathrm{MHz}$. Great care has been taken to the symmetry of the resulting pulses that should present sharp edges to be as close as possible to the ideal box problem. The intensity profile of these 52 ps flat-top pulses recorded with a high-speed photodiode is provided in the inset of Fig. 2. The resulting pulse is then amplified by an erbium doped fiber amplifier (EDFA). The peak-power of the pulse is accurately controlled by an optical variable attenuator. Note that contrary to experiments dealing with DSW generation in normally dispersive fibers $[14,25]$, the level of the extinction ratio is not here of crucial importance: in the focusing regime of propagation, a residual coherent background will not interfere with the pulse structure. On the contrary, one limiting factor could be the development of unwanted spontaneous modulation instability on the top of the super-Gaussian pulse $[19,20]$. As mentioned previously, to circumvent this problem, we have chosen a pulse with a relatively short duration and we inserted a narrow-band optical bandpass filter (OBPF) with a spectral width of $1 \mathrm{~nm}$. The propagation takes place in a standard single-mode fiber (SMF28) with parameters corresponding to the ones described previously. Different lengths of fibers ranging from $1 \mathrm{~km}$ up to $3.7 \mathrm{~km}$ have been tested during the experiments. The analysis of the output signal is carried out in the temporal and spectral domains by means of an optical sampling oscilloscope (OSO) and an optical spectrum analyzer (OSA), respectively.

In Fig. 3a, we report a first series of measurements according to the propagation distance. More precisely, thanks to a full set of SMF segments characterized by different lengths, we have depicted the output intensity profile as a function of the propagation distance for a fixed input peak power $P_{0}=1.9 \mathrm{~W}$. We clearly observe in the concatenated map the expanding nonlinear oscillatory regularization emerging from the initial discontinuous nature of the intensity profile.
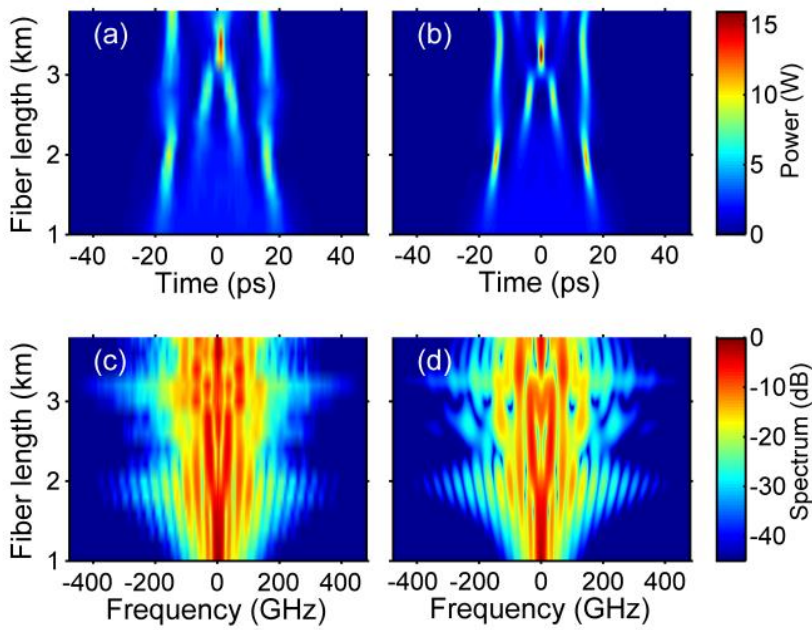

Fig. 3. Longitudinal evolution of (a) temporal and (c) spectral intensity profiles for $P_{0}=1.9 \mathrm{~W}$ recorded experimentally (b,d) Corresponding simulations based on the NLSE with fiber losses taken into account.

Indeed, after $1.5 \mathrm{~km}$ of propagation, two typical high-peak power spikes of light (FWHM duration of $\sim 2 \mathrm{ps}$ ) are generated in the edges of the super-Gaussian wave. Their temporal separation of $\sim 30 \mathrm{ps}$ is in agreement with the results expected from numerical simulations (see Fig. 3b) including typical losses of $0.2 \mathrm{~dB} \cdot \mathrm{km}^{-1}$. The ratio between the peak power of these spikes and the top of the initial super-Gaussian pulse is also well predicted. We can notice that, as expected by our numerical results, these structures emerge from the flattop edges of the pulse, subsequently compress and reach a maximum peak power before partly vanishing after $2.5 \mathrm{~km}$. Following this breathing stage, a second set of oscillations emerge in the inner part of the pulse and counter-propagate. This particular dynamics is one of the main features of the focusing dispersive dam break flows in the box problem. As a result, for longer propagation distance, a collision occurs to generate a giant spike here localized at $z=3.2 \mathrm{~km}$ [19]. The spectral evolution displayed in Fig. 3c confirms the complex dynamics involved by the super-Gaussian pulse in agreement with the simulations reported in Fig. 3d. The initially narrow 79-GHz spectrum ($20 \mathrm{~dB}$ spectral width) broadens up to a $\sim 420-\mathrm{GHz}$ width with a triangular shape (when plotted in a logarithmic scale, see Fig. 4c) that is typical of Peregrine breathers [2,3]. Due to the presence of two structures, the spectrum is intensity modulated with a period of $\sim 34-\mathrm{GHz}$ that corresponds to the inverse of the temporal spacing between the pulses (see also Fig. 4c). The breathing of nonlinear structures is also apparent on the spectrum map for longer propagation distances. The emergence 
of additional and unequally temporally spaced peaks leads to more complex spectra. We have checked that no spectral signature of unwanted spontaneous MI was visible in the spectrum.

In order to go further into the analysis of the generated highly localized structures, we have characterized their pulse shape at different stages of propagation and compare in Fig. 4 their intensity profiles with a genuine Peregrine breather solution of the NLSE. Details of the structure obtained at the first point of maximum compression is provided in Fig. 4a and outline a slight discrepancy regarding the peak-power (i.e., maximal focusing) that may be attributed to the bandwidth limitation of our OSO.
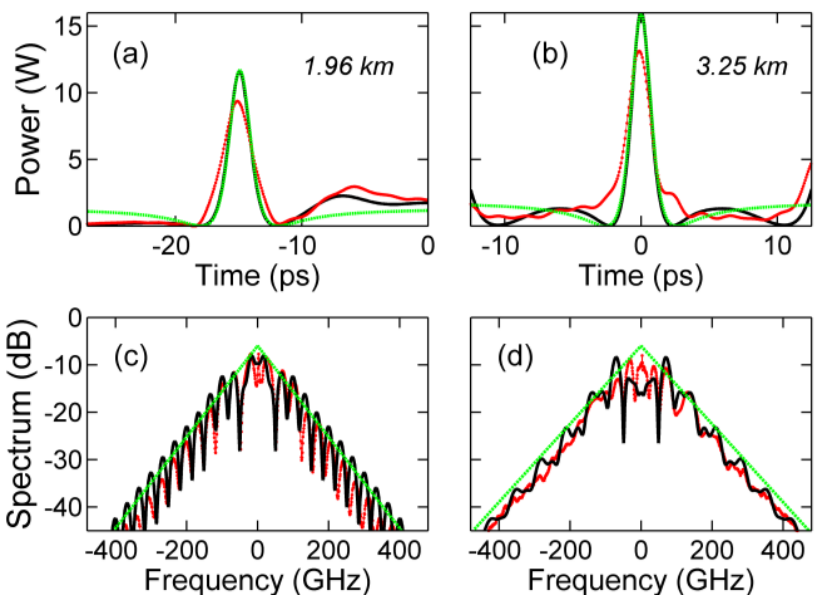

Fig. 4. (a,b) Temporal and (c,d) spectral intensity profiles obtained at two propagation distances 1.96 and $3.25 \mathrm{~km}$. Experiments are shown with magenta lines whereas simulations are shown with black lines. Theoretical fit: Peregrine breather solution (green dashed line).

Therefore, this limitation as well as a small temporal asymmetry in the input experimental waveform, also prevent us to clearly resolve the exact formation of the giant structure emerging at the collision of counter-propagating modulated wave trains $(z=3.25 \mathrm{~km})$ in Fig. 4b. Spectral measurements are summarized in Fig. 4c-d and show a good agreement with the numerical simulations.

To conclude, we have provided the experimental evidence of the complex nonlinear reshaping of a super-Gaussian pulse upon nonlinear propagation in a weakly dispersion focusing medium. Our results confirm the qualitative behavior measured in the spatial domain [20] and the power of the space/time duality. After an initial shock-like stage induced by the overlap of the highly chirped and sharp edges of the pulse with its top region, strong temporal oscillations appear and nonlinearly reshape into a Peregrine-like structure at each maximum compression. This transient evolution is then marked by the breathing of the wave structures. Contrary to Ref. [20], we have used fully coherent initial conditions and a medium of propagation with fully instantaneous nonlinear Kerr response. Finally, we have also characterized the collision of the two counter-propagating dispersive dam break flows in the form of a Peregrine-like structure.

Acknowledgments. We acknowledge Prof. Guy Millot for providing the super-Gaussian laser source.
Funding. Agence Nationale de la Recherche (ANR) (ANR-15-IDEX03, project PIA2/ISITE-BFC ; ANR-11-LABX-01-01, Labex Action); Région Bourgogne-Franche-Comté (PARI Photcom); Program FEDER-FSE Bourgogne 2014-2020; Institut Universitaire de France (IUF).

\section{References}

1. L. F. Mollenauer, R. H. Stolen, and J. P. Gordon, Phys. Rev. Lett. 45, 1095 (1980).

2. B. Kibler, J. Fatome, C. Finot, G. Millot, F. Dias, G. Genty, N. Akhmediev, and J. M. Dudley, Nat. Phys. 6, 790 (2010).

3. K. Hammani, B. Kibler, C. Finot, P. Morin, J. Fatome, J. M. Dudley, and G. Millot, Opt. Lett. 36, 2140 (2011).

4. B. Frisquet, B. Kibler, and G. Millot, Phys. Rev. X 3, 041032 (2013).

5. B. Frisquet, A. Chabchoub, J. Fatome, C. Finot, B. Kibler, and G. Millot, Phys. Rev. A 89, 023821 (2014).

6. B. Kibler, A. Chabchoub, A. A. Gelash, N. Akhmediev, and V. E. Zakharov, Phys. Rev. X 5, 041026 (2015).

7. A. Tikan, C. Billet, G. El, A. Tovbis, M. Bertola, T. Sylvestre, F. Gustave, S. Randoux, G. Genty, P. Suret, and J. M. Dudley, Phys. Rev. Lett. 119, 033901 (2017).

8. M. Närhi, B. Wetzel, C. Billet, S. Toenger, T. Sylvestre, J. -M. Merolla, R. Morandotti, F. Dias, G. Genty, and J. M. Dudley, Nat. Commun. 7, 13675 (2016).

9. P. Suret, R. El Koussaifi, A. Tikan, C. Evain, S. Randoux, C. Szwaj, and S. Bielawski, Nat. Commun. 7, 13136 (2016).

10. A. Chabchoub, N. P. Hoffmann, and N. Akhmediev, Phys. Rev. Lett. 106, 204502 (2011).

11. H. Bailung, S. K. Sharma, and Y. Nakamura, Phys. Rev. Lett. 107, 255005 (2011).

12. G.A. El and M. A. Hoefer. Physica D: Nonlinear Phenomena 333, 11 (2016)

13. J. Fatome, C. Finot, G. Millot, A. Armaroli, and S. Trillo, Phys. Rev. X 4, 021022 (2014).

14. G. Xu, M. Conforti, A. Kudlinski, A. Mussot, and S. Trillo, Phys. Rev. Lett. 118, 254101 (2017).

15. B. Wetzel, D. Bongiovanni, M. Kues, Y. Hu, Z. Chen, S. Trillo, J. M. Dudley, S. Wabnitz, and R. Morandotti, Phys. Rev. Lett. 117, 073902 (2016).

16. A. Chabchoub, B. Kibler, C. Finot, G. Millot, M. Onorato, J. M. Dudley, and A. V. Babanin, Ann. Phys. 361, 490 (2015).

17. G. B. Whitham, Linear and Nonlinear Waves (Wiley, New York, 1974).

18. R. Jenkins and K. D. McLaughlin, Commun. Pure Appl. Math. 67, 246-320 (2014).

19. G. A. El, E. G. Khamis, and A. Tovbis, Nonlinearity 29, 2798 (2016).

20. W. Wan, Dmitry V. Dylov, C. Barsi, and J. W. Fleischer, Opt. Lett. 35, 2819 (2010).

21. M. Bertola and A. Tovbis, Commun. Pure Appl. Math. 66, 678 (2013)

22. S. Randoux, P. Suret, G. El, Scientific reports 6, 29238, (2016)

23. G. P. Agrawal, Nonlinear Fiber Optics. Fifth Edition (Academic Press, 2013).

24. J. M. Dudley, G. Genty, F. Dias, B. Kibler, and N. Akhmediev, Opt. Express 17, 21497 (2009).

25. A. Parriaux, M. Conforti, A. Bendahmane, J. Fatome, C. Finot, S. Trillo, N. Picqué, and G. Millot, Opt. Lett. 42, 3044 (2017). 